\title{
The Opinions of Some Dental Hygienists about Improvement of Korean Dental Hygiene Education
}

\author{
Yang-Keum Han and Soo-Jeong Hwang ${ }^{1, \dagger}$ \\ Department of Dental Hygiene, Daejeon Health Institute of Technology, Daejeon 34504, \\ ${ }^{1}$ Department of Dental Hygiene, College of Medical Science, Konyang University, Daejeon 35365, Korea
}

This study's aim was to investigate their opinions to improve the educational system for dental hygienists on the basis of their duties as dental hygienists. The qualitative study was conducted among 19 dental hygienists at Daejeon, Korea from July to August 2016. Two researchers followed each subject for working hours in a day and recorded all the tasks and time to take for each task. After one day, the researchers met each subject and conducted face-to-face interviews to investigate the opinions about the dental hygiene curriculum and national board examination. The main duties recognized by more than half of the subjects were oral disease prevention including scaling and dental treatment assistance. The subjects' minority opinion about the main duties included radiography, impression taking, dental implant surgery assistance, orthodontic treatment, patient counseling, dental management and staff management, and oral health education. The most important tasks perceived by the subjects were prosthetic and implant impressions, scaling and implant surgery assistance. The subjects' minority opinion about the most important duties included patient counseling and making temporary crowns. The most difficult tasks answered by the subjects were prosthetic and implant impressions and dental implant surgery assistance. The subjects' minority opinion about it included patient counseling, scaling, and making temporary crowns. They mentioned that their curriculum in college was different from the actual work and the national board examination was not reflective of their real duties. We found out Korean dental hygienists had a lot of roles as dental assistants, dental business managers, and so on. We suggest that the law, curriculum and national board examination for dental hygienists should be revised to be able to reflect the reality of the clinical field.

Key Words: Curriculum, Dental hygienists, Korea, Licensure, Work

\section{Introduction}

Dental hygiene is the science of oral health and the prevention of oral disease ${ }^{1}$. Dental hygiene care plays a main role in the delivery of preventive care service to healthy people and of treatment to periodontitis patients ${ }^{2}$. The job of dental hygienists in Korea is prescribed in the law about medical technicians ${ }^{3}$. Dental hygienists' duties include preventing dental disease and taking intraoral radiographs in dental clinics under dentists' order according to the law. There has been no job to specialize in dental treatment assistance like dental assistants in Korea, although practical nurses can work in dental clinics. A lot of dental hygienists in Korea have assisted all kinds of dental treatments although these were not stipulated clearly by the $\operatorname{law}^{4,5)}$. Korean Dental Hygienists Association tried to revise the law to reflect the reality of dental hygienists' work. The law revised in 2011 included temporary filling, temporary prosthodontic cementation, removal of temporary fillings, impression taking and orthodontic wire ligation and removal ${ }^{6}$. Nonetheless, the considerable proportions of the duties that dental hygienists have performed in dental clinics previously could not be included in the law due to a lack of an agreement between a few interest groups including the Korean Dental Hygienists Association, Korean Dentist Association, Korean Licensed Practical Nurses Association, and so on.

Dental treatment has been constantly changing according 
to the development of medical technology, for example, dental implants, digital radiographs, and computer-aided design/computer-aided manufacturing (CAD/CAM) systems. A lot of Korean dental hygienists assist dental implant surgery and have higher workloads on dental implant surgery because Nation Health Insurance covers dental implant surgery for patients over 65 years old. Nonetheless, the items of the national board examination have not followed the rapid development of dental technology. The previous studies indicated that the national board examination had not properly reflected the real job of dental hygienists ${ }^{7-10}$. The curriculum of dental hygiene has focused on the subjects of the national board examination for dental hygienists, but little focus on the duties of dental hygienists ${ }^{11)}$. Cho ${ }^{12)}$ suggested that the curriculum of dental hygiene be changed from an academic-oriented curriculum to a duty-oriented curriculum. There was no obvious change to be detected in the curriculum of dental hygiene. Moreover, the law and national board examination being unable to be changed easily seem to be an obstacle to improving the curriculum of dental hygiene.

We consider the need to investigate the dental hygienists' present duties in dental fields in detail because the dental hygienists in Korea seemed to hesitate to talk about their duties in the clinic owing perhaps to the ambiguity of the legal duties and fear of illegality. We would like to show the difference between legal and actual work through an observational study and face-to-face interviews. In addition, we present the need to report their opinion about the dental hygiene curriculum and national board examination based on their real duties. This study's aim was to investigate the clinical duties of some dental hygienists in Korea and their opinions about education and national board examinations for dental hygienists to improve the educational system for dental hygienists and to revise the law for dental hygienists.

\section{Materials and Methods}

\section{Sampling}

This cross-sectional study was approved by the Institutional Review Board of Konyang University Hospital (KYUH 2016-01-015). Although the subjects were selected by convenience sampling, we tried to select the subjects to have representativeness as follows: The Daejeon Chungnam Dental Hygienists Association recommended some dental clinics and hospitals, considering the fields in which dental hygienists could work. We contacted the managers of the dental clinics and hospitals and explained the purpose of the study. If the subjects rejected this study, other dental clinics were recommended by the local dental hygienists association. One university hospital, one dental hospital, five dental clinics and one health center were selected. We planned to select seven dental hygienists with less than one year of job experience and seven with more than three years of job experience as the subjects of this observational study for one whole day. If an observational study was impossible for one day due to certain conditions of the selected dental clinics, other dental hygienists who do similar work were further selected. Finally, ten new dental hygienists and ten dental hygienists with a lengthier career gave their written consent to participate in this study, but one subject declined a face-to-face interview after the observational study. The dental hygienists included two in the university hospital, two in the dental hospital, two in the health center, and 13 in the dental clinics. The subjects in the university hospital were in charge of all dental treatment because the dental department of the university hospital was not comparatively large and had no specialty department. One in the dental hospital worked in the department of oromaxillofacial surgery, and the other in the dental hospital worked in the department of orthodontics. Two subjects who worked in the dental clinic specialized in orthodontic treatments. All were females.

\section{Observational study and face-to-face survey}

Two interviewers who had the license of dental hygienist visited the subjects' workplace, followed the subjects during their working hours in a day and recorded all the tasks of each dental hygienist and the time spent on each task. After one day, the interviewers conducted face-toface survey based on the observation records. To improve reliability, the research director discussed categorizing the tasks and questions included in the face-to-face survey with the researchers before and after the observational 
study. The questionnaire of the face-to-face interviews included queries regarding salary, clinical career, working hours, main duty, in-work training, perceived difficulty of the dental hygienists' job, opinions on the curriculum of the undergraduate school, opinions on the national examination of dental hygienists, and so on. We provided them the record of the observational study and the curriculum of the undergraduate school of each subject.

\section{Data description}

The criteria of the categorization in the observational data were the second report about the duties of Korean dental hygienists by the Korea Health Personnel Licensing Examination Institute. The categorization in interview data was revised on the basis of discussion with two interviewers and two research directors. The researchers categorized the results into their perception about the duties as dental hygienists and the opinions on dental hygiene education. The opinions on dental hygiene education were divided into dental hygiene curriculum and national board examination. Some of the face-to-face interviewers cited the subjects' answers as they were for conveying their feelings as a qualitative study. When one subject made multiple responses, they were considered as duplicate responses.

\section{Results}

\section{The clinical duties of the subjects through observational study}

The orders of the most frequent duties of 19 dental hygienists were as follows: infection control, management of treatment charts, protection of patients and dental staff, patient counseling, preparing treatment, oral health education, preparing intraoral or extraoral radiographs, scaling with ultrasonic scalers, assistance for orthodontic treatment, assistance for prosthetic treatment, assistance for conservative dental treatment, preparing infiltration anesthesia, dental equipment and device management, impression taking, teeth cleaning, orthodontic ligature, panorama taking, control of children patients, fluoride application, and intraoral radiographs taking.
The orders of the most time-consuming duties of 19 dental hygienists were as follows: infection control, patient counseling, scaling, documents management, preparing dental implant surgery, individual oral health education, assistance for orthodontic treatment, assistance for prosthetic treatment, management of dental equipment and unit-chair, assistance for conservative dental treatment, management of dental materials, patient management, assistance for endodontic treatment, alginate impression taking, assistance for periodontal treatment, rubber impression taking, adaptation and removal of prosthesis, ligaturing and removal of wire, control of children's behavior during treatment, temporary crown making, root planning, preparing intraoral or extraoral radiographs, panorama taking, preparing tooth extraction, tooth cleaning, uniform management, computed tomography taking, cephalometric radiograph taking, fluoride application, and periapical radiographs taking.

\section{The job recognition of the subjects}

Table 1 shows the work experience, working hours, annual salary, and main duties of 19 dental hygienists according to face to face survey. The major duty, the most important duty, and the most difficult duty in Table 1 were described as the subjects said. Fourteen subjects were working 40 hours a week and five were working 48 hours a week. Even if 12 subjects worked for 40 hours a week, they worked on every Saturday or every other Saturday instead of having a day off on weekdays. The annual salary after tax for the first year varied from 15 to 21.6 million Korean won.

The main duties recognized by more than half of the subjects were oral disease prevention including scaling and dental treatment assistance. The subjects' minority opinion about the main duties included radiography, impression taking, dental implant surgery assistance, orthodontic treatment, patient counseling, dental management and staff management, and oral health education. The most important tasks that were perceived subjectively were prosthetic and implant impressions, scaling and implant surgery assistance. The subjects' minority opinion about the most important duties included patient counseling and making temporary crowns. The most difficult tasks 


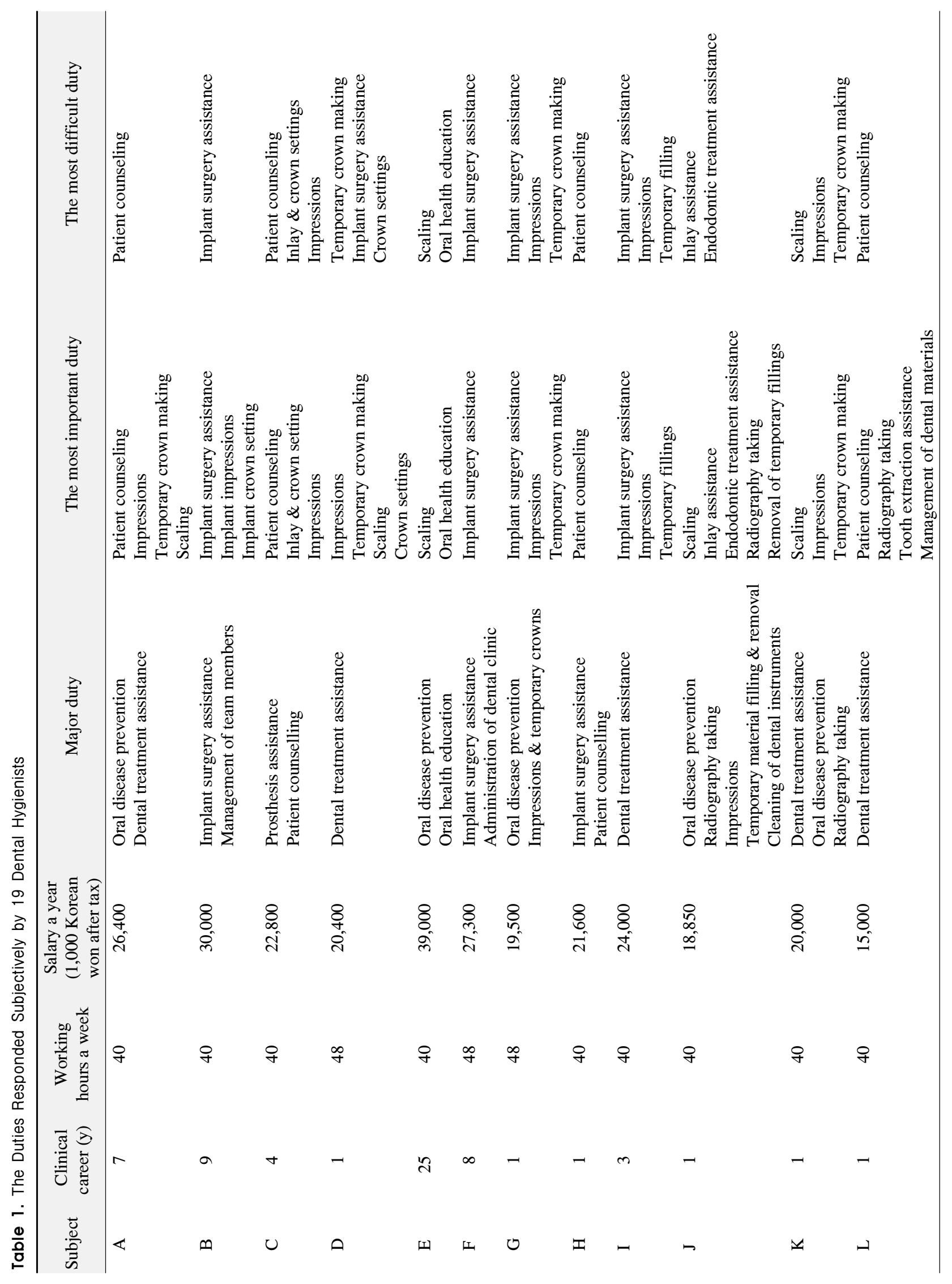




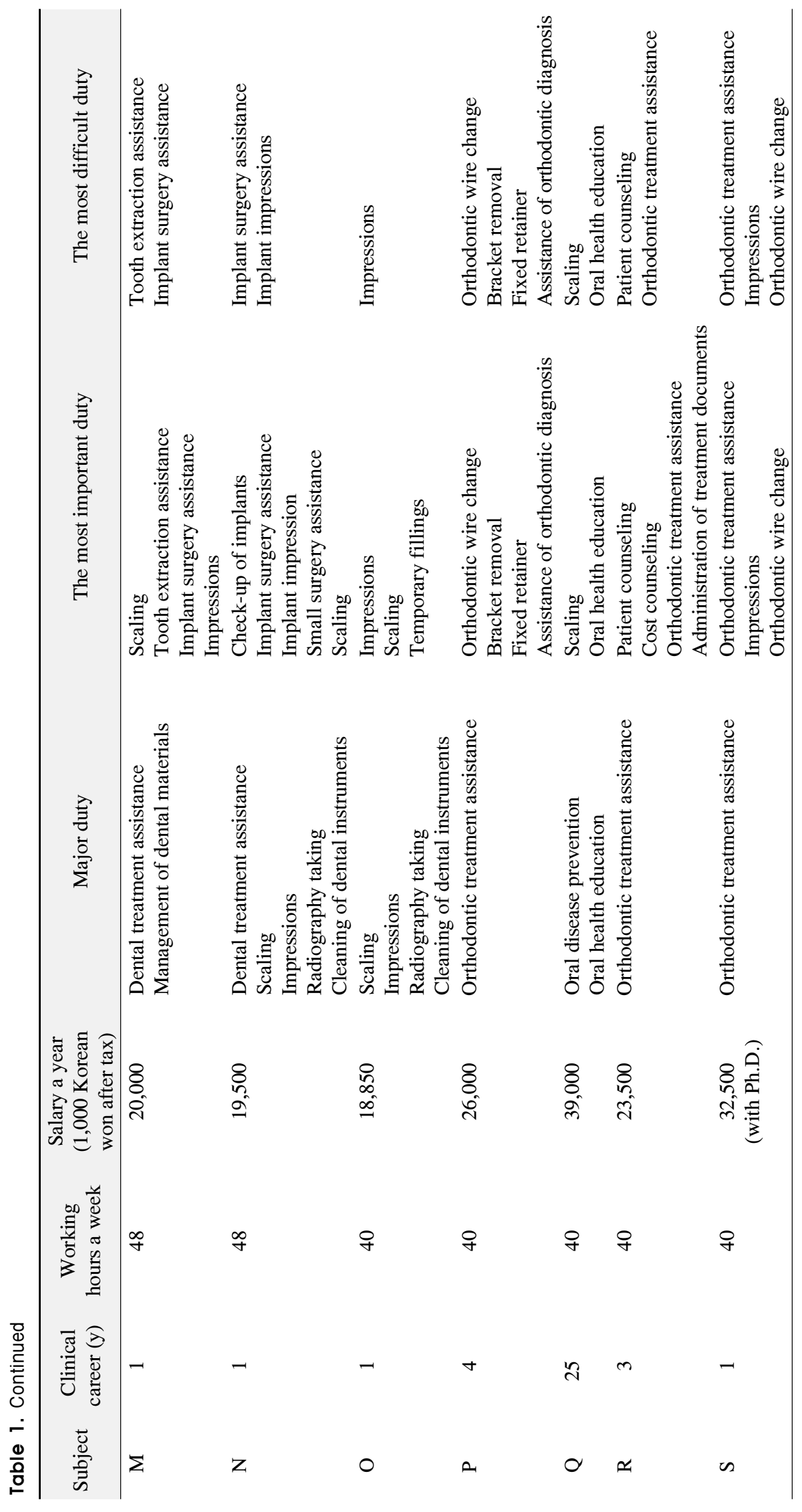


were prosthetic and implant impressions and dental implant surgery assistance. The subjects' minority opinion about the most difficult duties included patient counseling, scaling, and making temporary crowns. The selfdevelopment efforts mentioned by the subjects were preparing an examination for a claim of national health insurance, studying foreign languages, preparing to enter graduate school, acquiring dental treatment knowledge through books or the Internet, attendance at dental hygienist seminars, and job practice after working hours.

\section{Perceived job difficulties for the dental hygienists}

\section{1) Interpersonal relationship}

More than half dental hygienists mentioned that interpersonal relationships were the hardest at work. They answered that the relationship with patients was the most difficult.

"It is very difficult to deal with patients' complaints, even though it may be natural that problems arise in the relationship with the patient because dental hygienists occupy a large portion of dental treatment." (Subject $F$ )

"It was very difficult to adapt to the clinic system when I was the beginner. It is very difficult to respond to patients' complaints now." (Subject D)

"I think the emotional toll of dental hygienists is very severe. It is very hard to deal with patients with different requirements." (Subject $M$ )

"I think dental hygienist is a difficult job because of the service to the patients and high proficiency of work." (Subject $P$ )

A few subjects answered relationships with colleagues and with dentists was the most difficult.

"Conflicts with the dentist or staff does not make me feel proud as a dental hygienist." (Subject A)

"Some dentists ignore and scold us in front of patients. They have to change the attitude toward dental hygienists." (Subject C)

"Some dental hygienists with a career are too authoritative. It is very hard to communicate with them as a beginner." (Subject $K)$

\section{2) Shortage of manpower}

They answered that they had to do a lot of duties in the clinics. They indicated the main reason for the difficulty was the overburdened work due to a shortage of manpower, especially for dental hygienists. They said insufficient manpower in the dental clinic could give rise to serious patient complaints and overwork of dental staff.

"We need more dental hygienists to diminish overtime and overload." (Subject E)

"When we have a lot of patients, we get irritated easily and are very sensitive to each other. We need to have spare time and a rest area." (Subject L)

\section{3) Unclear legal duties}

They answered they had stress about unclear legal duties. They said some legal duties were described as too specific while others were described as vague. They sometimes felt anxious and distressed when they were working. They wanted to correct the law as it reflects the reality of clinical fields, not depending on its interpretation by the Ministry of Health and Welfare.

“Dental hygienists' legal duties are not clear, although we do a lot of work in dental clinics. The law about dental hygienists must be corrected according to the real job of dental hygienists." (Subject $C)$

"The dentists make us do a lot of duties in the dental clinic. We work with suspicion whether these are our duties or not." (Subject J)

"Subgingival calculus removal is not permitted as a job of Korean dental hygienists based on the interpretation by the Ministry of Health and Welfare, but it is included in the practical examinations of the national board examination. Can we do it or not?" (Subject D)

\section{The opinion about education for dental hygienists}

\section{1) Need for curriculum change of dental hygiene}

Most of them answered that the curriculum of dental hygiene needed to be changed to focus on clinical training rather than theoretical education. They wanted the curriculum to be about implant surgery assistance, digital radiographs taking, and National Health Insurance claims. The minority opinions were communication with patients, 
impression taking, use of new scaling devices, intramuscular injections, and oral health education as appropriate for each patient. Some said the rate of dental treatment assistance in the curriculum must increase, especially for dental implants. Some answered that the subject for conversation with foreigners in the dental clinic was needed. A few responses are as follows: professional ethics to represent rights and interests of dental hygienists, practical microbiology and immunology available in the clinic, and anatomy and physiology for treating patients with medical disease.

"The current curriculum focus is on academic education, so it is different from the real situation in dental clinics. Besides, the latest technology or machinery to use now is not included." (Subject A)

"Because digital radiographs devices are rapidly expanding in clinical markets, the curriculum needs to be followed by rapid change in dental treatment." (Subject J)

"Even though we meet so many cases of dental implants in dental clinics, we learned only a little bit about it." (Subject B)

"Although temporary crown making and impression taking are the high frequency task of Korean dental hygienists in dental clinic, we didn't learn the tasks sufficiently." (Subject I)

\section{2) Need for change in National Board Examination of Dental Hygienists}

All of them said to have to improve the National Board Examination of Dental Hygienists. Most of them indicated that the practical examination that focuses on only calculus detection and removal with hand instruments in two teeth in 4 minutes was not proper. Others said that the written test with the closed question to select the best answer had a limitation not to be able to reflect the real dental field.

"We sometimes use a hand scaler, and we always use an ultrasonic scaler in the clinical field. Nonetheless, we practiced using only hand instruments most of the time to pass the national board examination. To use an ultrasonic scaler should be considered in the national board examination." (Subject $H$ )

"Dental hygienists do a variety of things in the dental clinic. It is unfair to judge pass or fail for accurate detection and removal of calculus within 4 minutes in the practical examination." (Subject $Q$ )

"I think calculus detection and removal need to be excluded in the written test, because it is tested in the practical test." (Subject $O$ )

"We cannot trust the objectivity of the grader of the practical examinations. We felt a large deviation among the graders." (Subject K)

"The written test includes over 20 subjects. Many applicants gave up studying some subjects because they have a few questions. The national board examination has to change from subject-specific questions to integrated questions." (Subject G)

"The national board examination needs to be improved from the theoretical test to the practical test to reflect the reality of dental hygiene." (Subject $L$ )

\section{Discussion}

Most of the previous studies about the work of Korean dental hygienists was based on the written survey. We intended to investigate the real tasks of the dental hygienists in dental fields through the observational study. The result showed the most frequent task was infection control, the second was dental treatment assistance, the third was patient counseling, and the fourth was oral disease prevention. Kim et al. ${ }^{13)}$ said the main tasks of Korean dental hygienists in dental clinics were dental business management, oral disease prevention, assistance for dental treatment, and oral health education. Kim and $\mathrm{Kim}^{4)}$ as well as Kwon and $\mathrm{Lee}^{5)}$ reported that the main job of Korean dental hygienists in dental clinics was assistance for dental treatment. Similar to the observational study, the face-to-face survey showed more than half of the subjects answered that dental treatment assistance including implant surgery, orthodontic treatment and so on were the main tasks. Unlike Korean dental hygienists, the main duties of British dental hygienists were dental health education, dietary counseling, periodontal index probing, and fissure sealants ${ }^{14)}$.

The Korean dental hygienists felt conflicted about their job which is often occupied by dental treatment assistance 
in dental clinics, because the curriculum of dental hygiene and the national board examination focuses on prevention of dental disease and oral health education. Choi et al. ${ }^{15)}$ wrote that the difference of an expected task and practical task in dental assistance affected an employee's intention to act. Some subjects in this study suggested that the proportion of dental treatment assistance in the curriculum should increase to accurately reflect the clinical field. Some dental hygienists mentioned temporary crown making, implant surgery assistance, digital radiographs taking, and national health insurance claims had to be included in the curriculum. Moon and $\mathrm{Lim}^{16)}$ wrote that Korean dental hygienists needed competencies for dental intermediary-managers as follows: dental business management, treatment support, human resources management, communication and customer management, leadership, and self-control. Nevertheless, none of them has been leaned in the curriculum properly because they were not tested in the national board examination.

Most of the subjects said the curriculum and the national board examination have to be changed in accordance with practical tasks in dental fields. $\mathrm{Ko}$ and $\mathrm{Bae}^{7)}$ wrote that the dental hygienists thought that the current national practical examination were insufficient for assessing clinical practice ability and counseling techniques for dental hygiene intervention. Nam et al ${ }^{8)}$ showed that $85.4 \%$ of the dental hygienists answered that the national board examination was not adequate. Park ${ }^{9)}$ found that the distribution of the subjects on the national board examination was not appropriately reflecting the clinical needs. Kim et al. ${ }^{10)}$ said $66.9 \%$ of the subjects answered 'Yes' for the necessity of changing the current subjects of the Korean dental hygienists' licensing examinations.

About 5,000 dental hygienists are licensed through national board examination every year, and Korea has one of the highest ranking numbers of dental hygienists. Nonetheless, dentists and dental hygienists are suffering from a lack of dental hygienists. The considerable numbers of dental hygienists have stopped working or transferred. The reasons why dental hygienists were easily transferred were mentioned as interpersonal conflict, improper job demand, low income, lack of job autonomy, and job instability ${ }^{17)}$. Burnout incidence among dental hygienists was shown to be considerably high, and dental hygienists said relatively negative concerns about the variety of tasks when compared with other professionals ${ }^{18)}$. Yee at al. ${ }^{19)}$ said $28.2 \%$ of dental hygienists changed jobs for conflicts with dentists and $17.1 \%$ changed office locations for conflicts with staff. Won and $\mathrm{Oh}^{20)}$ said dental hygienists had more psychological stress than job stress. The subjects of this study said that the most difficult thing in their work was interpersonal relationships as well. Unlike previous studies which mention conflict with dentists or conflict with other staff ${ }^{4,15)}$, the subjects pointed out the relationship with patients as the hardest task. Recently, Korean patients tend to go around and shop for medical or dental clinics without trust for medical or dental professionals. Dental hygienists, the staff who communicate with patients, seem to have a higher burden in dental clinics.

We inspected the duties of some dental hygienists directly and heard their opinions in Korea. We found out that they thought there were the gaps between dental hygiene education and the clinical duties. They said that the curriculum was different from the actual work and the national board examination was not reflective of their real duties. We thought one of the reasons of the gaps might be the dental hygiene educational community that considered that dental treatment assistance was not the main duty of Korean dental hygienists. However, the subjects worked as dental hygienists and had a lot of roles such as dental assistants, dental business managers, radiologists, and dental technicians in the real fields. Although the dental hygiene educational community wants to embrace reality and change, the current law on dental hygienists is not supported to be able to do the real duties.

This study has the significance of being the first survey to observe the duties of dental hygienists directly in Korea, although they might have been influenced by the observer. We couldn't report each dental hygienist's duties because some duties could have been legal problems, although each duty needed to be reported through the observational study. We tried to show reality as the sum of the duties of each dental hygienist. We would conduct further studies of the opinions of dentists about the duties of dental hygienists, and the opinions of faculty in dental hygiene about dental hygiene curriculum and the national board 
examination based on this study. We suggest all occupations in the dental field need to discuss their work according to the situation in Korea and revise their laws to be able to reflect the real and clinical field. We also suggest the national examination committee and faculty council try to produce the questions that integrate several subjects in order to measure integrative thinking suited to the actual situation in Korea.

\section{References}

1. Daniel SJ, Harfst SA, Wilder RS: Mosby's dental hygiene. 2nd ed. Mosby-Elsevier, St. Louis, p.4, 2008.

2. Luciak-Donsberger C: Origins and benefits of dental hygiene practice in Europe. Int J Dent Hyg 1: 29-42, 2003. https://doi.org/10.1034/j.1601-5037.2003.00008.x

3. The law about medical technicians. Retrieved July 8, 2017, from http://www.law.go.kr/lsInfoP.do?lsiSeq=188096\&efYd= 20161202\#0000(2016, December 2).

4. Kim JH, Kim HJ: Relationship between turnover and job stress of dental hygienists. J Dent Hyg Sci 11: 47-53, 2011.

5. Kwon JY, Lee SY: Relationship of between task performance, job satisfaction, and organizational contribution of dental hygienists. J Dent Hyg Sci 16: 302-309, 2016. https://doi.org/10.17135/jdhs.2016.16.4.302

6. Act on the law about medical technicians. Retrieved July 8, 2017, from http://www.law.go.kr/lsInfoP.do?lsiSeq=187807\& efYd=20161130\#0000(2016, November 30).

7. Ko DK, Bae SS: The dental hygienists' perception of the national practical examination. J Dent Hyg Sci 16: 488-494, 2016. https://doi.org/10.17135/jdhs.2016.16.6.488

8. Nam YO, Jang HS, Kim YS: A study on improvement for national examination of dental hygienists in Korea. J Korean Soc Dent Hyg 10: 1-15, 2010.

9. Park MK: A survey on the perception of the national examination by dental hygienists. J Korean Acad Dent Hyg 13: 103-116, 2011.
10. Kim SH, Jang JH, Oh SH: A study for the improvement subjects of the Korean dental hygienists' licensing examination. J Dent Hyg Sci 9: 353-360, 2009.

11. Lee HS: Analysis of dental hygiene curriculum of dental hygiene programs in Korea. J Korean Soc Dent Hyg 9: 1-19, 2009.

12. Cho YS: Comparison of curriculums of dental hygiene education programs for B.S degree. J Dent Hyg Sci 5: 251-258, 2005.

13. Kim EG, Lim SH, Kwon MY, Choi YY, Han JH: Analysis of tasks and education needs for dental hygienist for development of dental hygiene curriculum. J Dent Hyg Sci 14: 35-42, 2014.

14. Gibbons DE, Corrigan M, Newton JT: A national survey of dental hygienists: working patterns and job satisfaction. $\mathrm{Br}$ Dent J 190: 207-210, 2001. https://doi.org/10.1038/sj.bdj.4800926

15. Choi HN, Bae HS, Cho YS: Effect of gap between expected job and performed job on turnover intention and job satisfaction in clinical dental hygienists. J Dent Hyg Sci 11: 463-469, 2011.

16. Moon HJ, Lim SR: Development of a job competency model of a dental intermediary-manager using the Delphi method. J Dent Hyg Sci 17: 150-159, 2017. https://doi.org/10.17135/jdhs.2017.17.2.150

17. Lee SY, Yu BC: Relationship between occupational stress and work-related factors in dental hygienists. J Dent Hyg Sci 11: 553-562, 2011.

18. Gorter RC: Work stress and burnout among dental hygienists. Int J Dent Hyg 3: 88-92, 2005. https://doi.org/10.1111/j.1601-5037.2005.00130.x

19. Yee T, Crawford L, Harber P: Work environment of dental hygienists. J Occup Environ Med 47: 633-639, 2005. https://doi.org/10.1097/01.jom.0000165745.89527.7e

20. Won YS, Oh HN: The effects of job stress and psychological stress among dental hygienists. J Dent Hyg Sci 15: 679-687, 2015. https://doi.org/10.17135/jdhs.2015.15.5.679 